\title{
Primary and secondary prevention of metabolic and cardiovascular comorbidities in women with polycystic ovary syndrome
}

\author{
Prevenção primária e secundária de co-morbidades metabólicas e \\ cardiovasculares em mulberes com síndrome do ovário policístico
}

Editorial

\section{Current concepts on polycystic ovary syndrome}

Polycystic ovary syndrome (PCOS) is a very common endocrine disease, affecting women of reproductive age. The prevalence of PCOS varies according to the diagnostic criteria used, with estimates ranging from $9 \%$ in women of reproductive age according to $\mathrm{NIH}$ criteria up to $18 \%$ with Rotterdam criteria $^{1,2}$.

Evidence indicates PCOS is a polygenic disease in which the individual susceptibility is also determined by environmental risk factors, including lifestyle. In essence, PCOS is characterized by androgen excess and chronic anovulation. However, the clinical presentation is heterogeneous among patients and may change in the same women along the years $^{2-4}$. Most common signs and symptoms include hirsutism, irregular menstrual cycles and infertility. In the last two decades it has become also clear that PCOS women often present insulin resistance and increased risk for metabolic syndrome, type 2 (T2) diabetes, dyslipidemia and hypertension.

Currently, the diagnosis of PCOS is confirmed according the Rotterdam Consensus ${ }^{5}$, an expansion of the former NIH criteria ${ }^{6}$. Proposed Rotterdam criteria for PCOS include two out of the following three: the presence of clinical and/or biochemical hyperandrogenism, oligomenorrhea/anovulation and the polycystic ovary appearance (PCO) on ultrasound. In turn, the Androgen Excess and PCOS Society ${ }^{7}$ considers that androgen excess is a central event in the pathogenesis and development of PCOS and that this criterion should be present for the diagnosis of PCOS. In any case, other androgen excess disorders, such as non-classic congenital adrenal hyperplasia (NC-CAH), Cushing's syndrome, androgen secreting tumors, hyperprolactinemia, thyroid diseases, drug-induced androgen excess should be excluded as well as the other causes for oligomenorrhea or anovulation ${ }^{5-7}$.

Recently, the Expert Panel from a NIH Evidence Based Methodology Workshop on PCOS reinforced the use of the wider Rotterdam criteria ${ }^{8}$. In consequence, new phenotypes had arisen in addition to the classic phenotype, in which patients present hyperandrogenism and oligomenorrhea with or without PCO on ultrasound. These new phenotypes are the

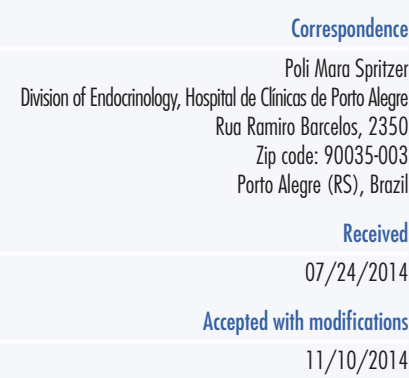

DO1: $10.1590 / 50100-720320140005212$
'Laboratory of Molecular Endocrinology, Department of Physiology, Universidade Federal do Rio Grande do Sul - UFRGS - Porto
Alegre (RS), Brazil. Conflict of interests: none. 
"ovulatory phenotype", that means hyperandrogenism and PCO in an ovulatory woman and the "non-hyperandrogenic phenotype", in which there is oligomenorrhea and PCO, without overt hyperandrogenism ${ }^{2,5,8}$.

Concerning ovarian morphology, PCO has been defined as an ovarian volume higher than $10 \mathrm{~cm}^{3}$ and/or the presence of multiple small follicles ${ }^{5}$. In this sense, with the recent improvements on ovarian imaging the current follicle number threshold value is being revaluated and will probably be increased from the prior follicle number of 12 or more follicles of $2-9 \mathrm{~mm}^{9-11}$.

\section{Metabolic disturbances and cardiovascular risk factors in women with polycystic ovary syndrome}

Obesity is a common characteristic of PCOS with a prevalence of around $50 \%$ according to different populations $^{12}$ and may exacerbate the metabolic and reproductive disorders associated with the syndrome ${ }^{13-15}$. In addition, PCOS women present higher risk for $\mathrm{T} 2$ diabetes and metabolic syndrome and sub-clinical atherosclerosis in $^{9}, 18$ (Figure 1) ${ }^{19}$. Metabolic syndrome is defined, for women, by the presence of three out of five criteria: waist circumference $>88 \mathrm{~cm}$, fast glucose $>100 \mathrm{mg} / \mathrm{dL}$, systolic blood pressure $>130$ and/or diastolic blood pressure $>85 \mathrm{mmHg}$, $\mathrm{HDL}-\mathrm{c}<50 \mathrm{mg} / \mathrm{dL}$ and triglycerides $>150 \mathrm{mg} / \mathrm{dL}^{20}$. Interestingly, despite presenting higher prevalence of cardiovascular risk factors, definitive evidence for increased incidence of cardiovascular events later in life is lacking in the PCOS population. Studies with small sample sizes, relatively short periods of follow-up, highly selected clinic populations, potential confounding due to effects of treatment at baseline and changing clinical phenotype over time are some reasons why further strict long-term prospective studies focusing on cardiovascular outcomes and clinical trials assessing changes on risk factors are needed in women with PCOS ${ }^{21}$.

Likewise, it has been shown that insulin resistance and their clinical consequences are more prevalent in PCOS women with the classic phenotype and to a lesser extent with the ovulatory phenotype ${ }^{16,22,23}$. In turn, the non-hyperandrogenic phenotype seems to function as a separate group, being metabolically similar to non-PCOS women ${ }^{8,23}$.

\section{Primary and secondary prevention of} metabolic and cardiovascular comorbidities in women with polycystic ovary syndrome

Beyond the management of hirsutism and reproductive abnormalities, concerns arise regarding how to prevent and treat prevalent metabolic and cardiovascular comorbidities in PCOS women. In this context, it is important to underline the central role of insulin resistance on the pathophysiology of metabolic syndrome and on cardiovascular risk in these women. However, while being a common trait, insulin resistance is not a universal feature of PCOS ${ }^{19,24}$. In addition, the focus of treatment should be their clinical consequences instead of the insulin resistance per se, such as metabolic syndrome, abdominal adiposity (waist circumference higher than $88 \mathrm{~cm}$ ) as well as impaired fasting glucose (IFG, fasting glucose between 100 and $125 \mathrm{mg} / \mathrm{dL}$ ), impaired glucose tolerance (IGT, $2 \mathrm{~h}$ post-glucose between 140 and $199 \mathrm{mg} / \mathrm{dL}$ ) and T2

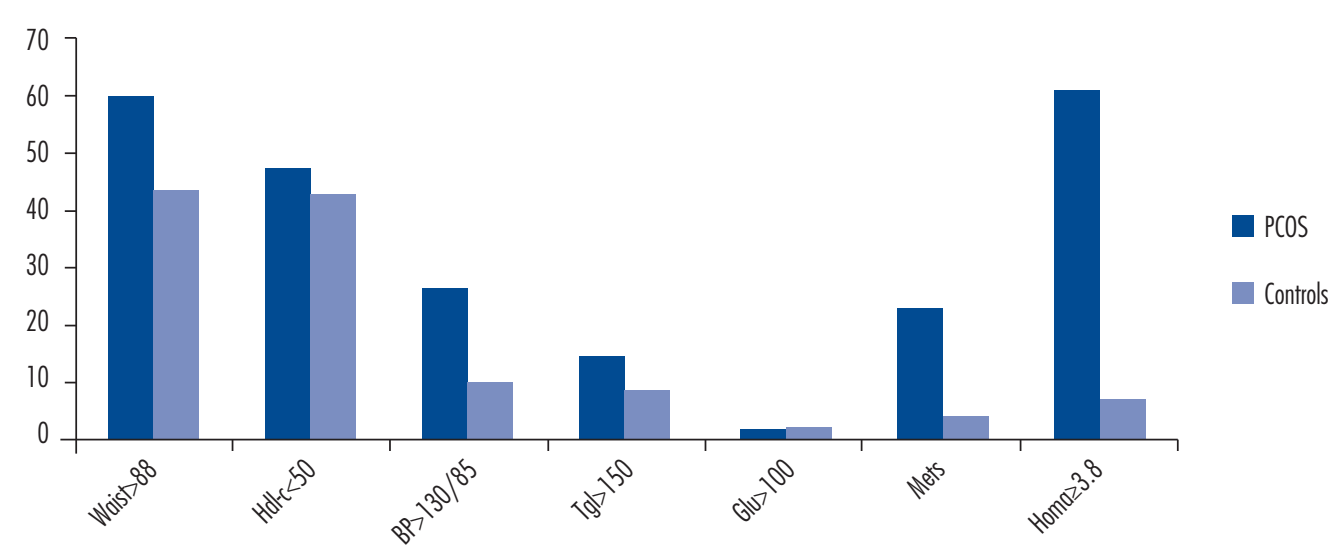

HDL-c: High-density lipoprotein cholesterol; BP: blood pressure; Tgl: triglycerides; Glu: fast glucose; MetS: metabolic syndrome; Homa: homeostatic model assessment of insulin resistance; PCOS: polycystic ovary syndrome. Adapted from: Spritzer and Wiltgen ${ }^{19}$

Figure 1. Prevalence of insulin resistance, metabolic syndrome and their isolated components in 91 women with polycystic ovary syndrome and controls, paired by age and body mass index. 
diabetes (fasting glucose equal or higher than 126 and/or $2 \mathrm{~h}$ post-glucose equal or higher than $200 \mathrm{mg} / \mathrm{dL})^{2,24,25}$.

Lifestyle change is the first-line non-pharmacological therapy to lessen cardiovascular and metabolic risks, mainly in overweight or obese PCOS women, and should combine behavioral (reduction of psychosocial stressors), dietary, and exercise management ${ }^{26-28}$. Lifestyle modification has been already proved to be effective in reducing the incidence of diabetes in persons at high risk ${ }^{29}$.

Nevertheless, in many cases it will be necessary to add a drug to lifestyle change. Pharmacological therapy should address specific goals, such as to treat dyslipidemia, metabolic syndrome, pre-diabetes or T2 diabetes, high blood pressure or severe obesity with statins, insulinsensitizing, antihypertensive and anti-obesity agents, respectively. Bariatric surgery is an additional option for PCOS women with severe obesity or obesity with metabolic comorbidities ${ }^{2,9}$.

The most widely insulin-sensitizing agent used for women with PCOS presenting metabolic syndrome, obesity or impaired glucose tolerance is metformin, in great part because of its impact on diabetes prevention in pre-diabetic subjects ${ }^{30}$. Besides its actions on glucose balance, reducing hepatic glucose output and increasing peripheral insulin action, metformin also directly inhibits ovarian androgen secretion ${ }^{2,31}$. In line with the current knowledge, metformin seems to minimize cardiovascular and metabolic risks in PCOS by improving insulin sensitivity, lowering blood glucose and androgen levels $s^{2,28,32}$. These effects are stronger if combined with lifestyle intervention ${ }^{2,28}$ and appear to be dose dependent, although this issue is not completely consensual ${ }^{33,34}$. While metformin is not effective to treat hirsutism it can improve menstrual disturbances and has been regarded as a second-line treatment for women with PCOS with contraindications for using contraceptive pills ${ }^{9,35}$.

Therefore, primary and secondary prevention of metabolic and cardiovascular comorbidities in PCOS involves firstly, an accurate and individualized clinical assessment of any PCOS woman. Obesity or normalweight with familiar history of diabetes, presence of clinical manifestations of insulin resistance such as acanthosis nigricans or central adiposity and the screening for metabolic and cardiovascular risk factors, mainly hypertension, dyslipidemia or dysglicemia (IFG, ITG or T2 diabetes), are clearly recommended. Secondly, according to the risk profile, treatment goals should be tailored to each individual PCOS women, covering both metabolic risks as well their main complaints related to menstrual disturbances, hirsutism or infertility.

\section{References}

1. March WA, Moore VM, Willson KJ, Phillips DI, Norman RJ, Davies $\mathrm{M}$. The prevalence of polycystic ovary syndrome in a community sample assessed under contrasting diagnostic criteria. Hum Reprod. 2010;25(2):544-51.

2. Spritzer PM. Polycystic ovary syndrome: reviewing diagnosis and management of metabolic disturbances. Arq Bras Endocrinol Metabol. 2014;58(2):182-7.

3. Azziz R, Woods KS, Reyna R, Key TJ, Knochenhaver ES, Yildiz BO. The prevalence and features of the polycystic ovary syndrome in an unselected population. J Clin Endocrinol Metab. 2004;89(6):2745-9.

4. Brown ZA, Louwers YV, Fong SL, Valkenburg $O$, Birnie $E$, de Jong $\mathrm{FH}$, et al. The phenotype of polycystic ovary syndrome ameliorates with aging. Fertil Steril. $2011 ; 96(5): 1259-65$.

5. Rotterdam ESHRE/ASRM-Sponsored PCOS Consensus Workshop Group. Revised 2003 consensus on diagnostic criteria and longterm health risks related to polycystic ovary syndrome. Fertil Steril. 2004;81(1):19-25.

6. Zawadski JF, Dunaif A. Diagnostic criteria for polycystic ovary syndrome: towards a rational approach. In: Dunaif A, Givens JR, Haseltuine F, editors. Polycystic ovary syndrome. Boston: Blackwell Scientific; 1992. p. 377-84.

7. Azziz R, Carmina E, Dewailly D, Diamanti-Kandarakis E, EscobarMorreale HF, Futterweit W, et al. Positions statement: criteria for defining polycystic ovary syndrome as a predominantly hyperandrogenic syndrome: an Androgen Excess Society guideline. J Clin Endocrinol Metab. 2006;91(1 1):4237-45.
8. National Institutes of Health. Evidence-based methodology workshop on Polycystic Ovary Syndrome. Workshop of the NIH Office Disease Prevention; 2012 Dec 3-5; Bethesda: NIH; 2012. [cited 2014 Nov 14]. Available from: <https://prevention.nih. gov/docs/programs/pcos/FinalReport.pdf>

9. Fauser BC, Tarlatzis BC, Rebar RW, Legro RS, Balen AH, Lobo $R$, et al. Consensus on women's health aspects of Polycystic Ovary Syndrome (PCOS): the Amsterdam ESHRE/ASRMSponsored 3rd PCOS Consensus Workshop Group. Fertil Steril. 2012;97(1):28-38.e25.

10. Dewailly D, Gronier H, Poncelet E, Robin G, Leroy M, Pigny P, et al. Diagnosis of polycystic ovary syndrome (PCOS): revisiting the threshold values of follicle count on ultrasound and of the serum $\mathrm{AMH}$ level for the definition of polycystic ovaries. Hum Reprod. $2011 ; 26(11): 3123-9$.

11. Lujan ME, Jarrett BY, Brooks ED, Reines JK, Peppin AK, Muhn N, et al. Updated ultrasound criteria for polycystic ovary syndrome: reliable thresholds for elevated follicle population and ovarian volume. Hum Reprod. 2013;28(5):1361-8.

12. Lim SS, Davies M, Norman RJ, Moran L. Overweight, obesity and central obesity in women with polycystic ovary syndrome: a systematic review and meta-analysis. Hum Reprod Update. 2012;18(6):618-37.

13. Diamanti-Kandarakis E, Spritzer PM, Sir-Petermann T, Motta AB. Insulin resistance and polycystic ovary syndrome through life. Curr Pharm Des. 2012;18(34):5569-76. 
14. Wild RA, Rizzo M, Clifton S, Carmina E. Lipid levels in polycystic ovary syndrome: systematic review and meta-analysis. Fertil Steril. $2011 ; 95(3): 1073-9 . e 1-11$.

15. Apridonidze T, Essah PA, luorno M, Nestler JE. Prevalence and characteristics of the metabolic syndrome in women with polycystic ovary syndrome. J Clin Endocrinol Metab. 2005;90(4):1929-35.

16. Wiltgen D, Spritzer PM. Variation in metabolic and cardiovascular risk in women with different polycystic ovary syndrome phenotypes. Fertil Steril. 2010;94(6):2493-6.

17. Di Domenico K, Wiltgen D, Nickel FJ, Magalhães JA, Moraes RS, Spritzer PM. Cardiac autonomic modulation in polycystic ovary syndrome: does the phenotype matter? Fertil Steril. 2013;99(1):286-92.

18. Nácul AP, Andrade CD, Schwartz P, de Bittencourt Jr PI, Spritzer PM. Nitric oxide and fibrinogen in polycystic ovary syndrome: associations with insulin resistance and obesity. Eur J Obstet Gynecol Reprod Biol. 2007;133(2):191-6.

19. Spritzer PM, Wiltgen D. [Prevalence of metabolic syndrome in patients of south of Brazil with polycystic ovary syndrome (PCOS)]. Arq Bras Endocrinol Metabol. 2007;51 (1):146-7. Portuguese.

20. Alberti KG, Zimmet P, Shaw J. Metabolic syndrome-a new worldwide definition. A Consensus Statement from the International Diabetes Federation. Diabet Med. 2006;23(5):469-80.

21. Cussons AJ, Stuckey BG, Watts GF. Cardiovascular disease in the polycystic ovary syndrome: new insights and perspectives. Atherosclerosis. 2006;185(2):227-39.

22. Yildiz BO, Bozdag G, Yapici Z, Esinler I, Yarali H. Prevalence, phenotype and cardiometabolic risk of polycystic ovary syndrome under different diagnostic criteria. Hum Reprod. 2012;27(10):3067-73.

23. Moghetti P, Tosi F, Bonin C, Di Sarra D, Fiers T, Kaufman JM, et al. Divergences in insulin resistance between the different phenotypes of the polycystic ovary syndrome. J Clin Endocrinol Metab. 2013;98(4):E628-37.

24. Marshall JC, Dunaif A. Should all women with PCOS be treated for insulin resistance? Fertil Steril. 2012;97(1):18-22.

25. Salpeter SR, Buckley NS, Kahn JA, Salpeter EE. Meta-analysis: metformin treatment in persons at risk for diabetes mellitus. Am J Med. 2008;121(2):149-57.e2.
26. Karimzadeh MA, Javedani $M$. An assessment of lifestyle modification versus medical treatment with clomiphene citrate, metformin, and clomiphene citrate-metformin in patients with polycystic ovary syndrome. Fertil Steril. 2010;94(1):216-20.

27. Graff SK, Mário FM, Alves BC, Spritzer PM. Dietary glycemic index is associated with less favorable anthropometric and metabolic profile in polycystic ovary syndrome women with different phenotypes. Fertil Steril. 2013;100(4):1081-8.

28. Nieuwenhuis-Ruifrok AE, Kuchenbecker WK, Hoek A, Middleton $P$, Norman RJ. Insulin sensitizing drugs for weight loss in women of reproductive age who are overweight or obese: systematic review and meta-analysis. Hum Reprod Update. 2009;15(1):57-68.

29. Knowler WC, Barrett-Connor E, Fowler SE, Hamman RF, Lachin $J M$, Walker EA, et al. Reduction in the incidence of type 2 diabetes with lifestyle intervention or metformin $\mathrm{N}$ Engl J Med. 2002;346(6):393-403.

30. Salpeter SR, Buckley NS, Kahn JA, Salpeter EE. Meta-analysis: metformin treatment in persons at risk for diabetes mellitus. Am J Med. 2008;121(2):149-57.

31. Attia GR, Rainey WE, Carr BR. Metformin directly inhibits androgen production in human thecal cells. Fertil Steril. 2001;76(3):517-24.

32. Ladson G, Dodson WC, Sweet SD, Archibong AE, Kunselman AR, Demers $L M$, et al. The effects of metformin with lifestyle therapy in polycystic ovary syndrome: a randomized double-blind study. Fertil Steril. $2011 ; 95(3)$ :1059-66.e 1-7.

33. Bruno RV, de Avila MA, Neves FB, Nardi AE, Crespo CM, Carneiro Sobrinho AT. Comparison of two doses of metformin (2.5 and 1.5 $\mathrm{g} /$ day) for the treatment of polycystic ovary syndrome and their effect on body mass index and waist circumference. Fertil Steril. 2007;88(2):510-2.

34. Fulghesu AM, Romualdi D, Di Florio C, Sanna S, Tagliaferri V, Gambineri $A$, et al. Is there a dose-response relationship of metformin treatment in patients with polycystic ovary syndrome? Results from a multicentric study. Hum Reprod. 2012;27(10):3057-66.

35. Costello M, Shrestha B, Eden J, Sjoblom P, Johnson N. Insulinsensitising drugs versus the combined oral contraceptive pill for hirsutism, acne and risk of diabetes, cardiovascular disease, and endometrial cancer in polycystic ovary syndrome. Cochrane Database Syst Rev. 2007;(1):CD005552. 